\title{
Checklist de Fanniidae (Insecta, Diptera) do Estado de Mato Grosso do Sul
}

\author{
Diana Grisales \& Claudio J. B. de Carvalho
}

Laboratório de Biodiversidade e Biogeografia de Diptera, Departamento de Zoologia, UFPR, Caixa Postal 19020, Curitiba, PR, Brasil, 81531-980. (cjbcarva@ufpr.br)

Recebido 23 novembro 2016

Aceito 6 fevereiro 2017

DOI: $10.1590 / 1678-4766 e 2017138$

ABSTRACT. Checklist of Fanniidae (Insecta, Diptera) of the State of Mato Grosso do Sul. A checklist of the Fanniidae species in the state of Mato Grosso do Sul, Brazil, is provided. There are 11 species recorded, which represent $21 \%$ of all Fanniidae species known in Brazil.

KEYWORDS. biodiversity, catalogue, little house fly, lesser house fly, Biota-MS Program.

RESUMO. Nesse artigo é fornecida uma lista de espécies de Fanniidae do estado de Mato Grosso do Sul. Até o momento são registradas 11 espécies, o que representa $21 \%$ das espécies de Fanniidae conhecidas para o Brasil.

PALAVRAS-CHAVE. biodiversidade, catálogo, mosca doméstica pequena, taxonomia, Programa Biota-MS.

Fanniidae é uma pequena família de Diptera com apenas cinco gêneros conhecidos no mundo. No Brasil ocorrem espécies de Euryomma Stein e Fannia RobineauDesvoidy, algumas bem comuns. São moscas de tamanho pequeno a médio, com o comprimento do corpo variando de 3-8 mm, geralmente de coloração escura ou acinzentada e com asa hialina ou maculada. Os adultos das espécies podem ser reconhecidos pelas calípteras desenvolvidas e ausência de cerdas no mero. Esses caracteres estão presentes nas espécies das famílias próximas, como Muscidae e Anthomyiidae. Entretanto, as espécies de Fanniidae segregam-se das de grupos próximos por características peculiares da venação da asa, como a subcostal não sinuosa e o formato sigmoide da veia $\mathrm{A}_{2}$ (CARVAlHo et al., 2002a). Os Fanniidae são frequentemente denominados de mosca doméstica pequena, pelo menor comprimento de Fannia canicularis (Linnaeus, 1761) em relação à Musca domestica Linnaeus, 1758, a mosca doméstica comum. Aparentemente, a cosmopolita $F$. canicularis seguiu o homem no mundo, da mesma maneira do que a M. domestica (Chillcott, 1961).

A biologia das espécies é pouco conhecida. Os adultos normalmente frequentam matéria orgânica em decomposição, possuindo, no entanto, várias espécies de hábito diverso, incluindo coprófago, como $F$. scalaris (Fabricius), a mosca das latrinas (CARVALHO et al., 2003). Esta espécie pode causar diversos tipos de míases intestinais no homem e seus animais domésticos (ChillcotT, 1961). A morfologia externa das larvas da Fanniidae se assemelha a larvas de Platypezidae e Phoridae (CourI, 1992). Representantes dessas famílias são conhecidos por se desenvolverem em fungos, o habitat mais comum das larvas de Fanniidae (Chillcott, 1961).

\section{MATERIAL E MÉTODOS}

Para a elaboração dessa lista de espécies, foram consultadas publicações com registros de espécies de Fanniidae para o estado de Mato Grosso do Sul: AlbuQUerQue et al., 1981; CAMPos \& Barros, 1995; Carvalho et al., 2002a; Gomes et al., 2002; Wendt \& Carvalho, 2009, assim como o mais recente Catálogo de Fanniidae da Região Neotropical (CARVALHO et al., 2003). Para completar a lista, foi consultada a Coleção Entomológica Padre Jesus Santiago Moure do Departamento de Zoologia da Universidade Federal do Paraná (DZUP).

\section{RESULTADOS E DISCUSSÃO}

Lista de espécies de Fanniidae do Estado de Mato Grosso do Sul. Das 52 espécies registradas para o Brasil, 11 espécies de Fannia (21\%) ocorrem no estado de Mato Grosso do Sul (CARvalho et al., 2003) (Tab. I).

A maioria do material registrado para o Mato Grosso do Sul vem de projetos específicos de coleta realizados pelo Serviço Nacional de Febre Amarela e o Instituto Oswaldo Cruz na década de 1930 e 1940 (AlbuQuerque et al., 1981). Da mesma forma, estudos para o conhecimento das espécies sinantrópicas e de importância econômica na região (veiculadoras de ovos da mosca do berne), contribuíram com parte da informação da biodiversidade de Fanniidae (ver GoMes et al., 2002; CARVALHO et al., 2002a). A maioria das 
Tab. I. Espécies de Fanniidae registradas para o estado de Mato Grosso do Sul, Brasil. Dados baseados em material examinado e referências bibliográficas: 1, Albuquerque et al., 1981; 2, Gomes et al., 2002; 3, Campos \& Barros, 1995; 4, Carvalho et al., 2002a; 5, Carvalho et al., 2003; 6, Wendt \& Carvalho, 2009; 7, Coleção Entomológica Padre Jesus Santiago Moure (DZUP).

\begin{tabular}{lll}
\hline Espécie & Referências & Localidades \\
\hline Fannia bahiensis Albuquerque, 1957 & $1,2,5$ & Campo Grande, Maracaju \\
Fannia bella Albuquerque, 1954 & 1,5 & Dourados \\
Fannia flavicincta (Stein, 1904) & 1,5 & Bodoquena \\
Fannia heydenii (Wiedemann, 1830) & $1,2,5,6,7$ & Bodoquena, Campo Grande, Maracaju \\
Fannia hirtifemur (Stein, 1904) & 1 & Maracaju \\
Fannia inermipennis Albuquerque, 1954 & 1 & Bodoquena, Salobra (localidade-tipo) \\
Fannia longipila Albuquerque, 1954 & $2,5,7$ & Campo Grande \\
Fannia obscurinervis (Stein, 1900) & 6 & desconhecida \\
Fannia petrocchiae Shannon \& Del Ponte, 1926 & 1,5 & Maracaju \\
Fannia pusio (Wiedemann, 1830) & $1,2,4,5,6,7$ & Campo Grande, Corumbá, Salobra \\
Fannia tumidifemur Stein, 1911 & $1,5,6$ & Bodoquena, Maracaju, Salobra \\
\hline
\end{tabular}

espécies registradas para a área é considerada sinantrópica e foi coletada principalmente em áreas urbanas e rurais ou com forte influência antrópica. Embora a economia do Mato Grosso do Sul esteja baseada na agricultura e na criação de gado e seja importante o conhecimento das espécies de Fanniidae que possam afetar estas atividades, o estado possui grandes áreas de floresta (e.g. Pantanal) que precisam ser amostradas, com o objetivo de conhecer melhor a biodiversidade, incluindo a de Fanniidae. Nas regiões oeste e centro do estado estão concentrados os maiores esforços de amostragem, demonstrando que ainda existe uma grande extensão do território por ser explorada.

Os principais grupos de pesquisa e coleções de Fanniidae no Brasil. Os principais grupos se encontram no Laboratório de Diptera do Museu Nacional do Rio de Janeiro (MNRJ) e no Laboratório de Biodiversidade e Biogeografia de Diptera do Departamento de Zoologia na Universidade Federal do Paraná (UFPR). Os acervos com maior número de espécies depositadas são a coleção Entomológica do Museu Nacional do Rio de Janeiro (MNRJ), a Coleção Entomológica do Museu Padre Jesus Santiago Moure do Departamento de Zoologia da Universidade Federal do Paraná (DZUP), a Coleção Entomológica do Museu de Zoologia da Universidade de São Paulo (USP) e a Coleção Entomológica do Instituto Nacional de Pesquisas da Amazônia (INPA) (CARVAlHo et al., 2002b).

Principais lacunas do conhecimento. As principais lacunas do conhecimento estão relacionadas à diversidade de espécies em regiões com pouca ou nenhuma amostragem, como as regiões Norte, Nordeste e Centro-Oeste do Brasil. No caso de Mato Grosso do Sul são necessárias coletas exaustivas com o objetivo de aumentar o conhecimento da biodiversidade de Fanniidae, tanto em áreas rurais quanto em áreas naturais. Além de conhecer a diversidade taxonômica que ocorre na região, também são necessárias coletas para a realização de estudos de ciclos de vida, descrições de imaturos e análises moleculares que permitirão a associação de sexos. Fêmeas de diferentes espécies de Fannia são muito semelhantes entre si, dificultando a identificação precisa.
Perspectivas de pesquisa para os próximos 10 anos. As perspectivas de pesquisa em Fanniidae passam por projetos de levantamentos faunísticos, com o do projeto Sisbiota para o Cerrado e Pantanal, áreas pouco amostradas.

Agradecimentos. A Fundação de Apoio ao Desenvolvimento do Ensino, Ciências e Tecnologia do Estado de Mato Grosso do Sul (Fundect) e a Superintendência de Ciências e Tecnologia do Estado de Mato Grosso do Sul (Sucitec/MS) pelo convite de participação neste fascículo especial da Iheringia, Série Zoologia e o suporte financeiro para sua publicação. A Kistern Lica F. Haseyama pela leitura, críticas e sugestões. D. G. é bolsista de Pós-doutorado júnior CNPq (PDJ) e CJBC é bolsista do CNPq (processo número 3047/2011-2).

\section{REFERÊNCIAS BIBLIOGRÁFICAS}

Albuquerque, D. de O.; Pamplona, D. \& Carvalho, C. J. B. De. 1981. Contribuição ao conhecimento dos Fannia R. D., 1830 da Região Neotropical (Diptera, Fanniidae). Arquivos do Museu Nacional 56:9-34.

Campos, C. F. M. \& Barros, A. T. M. 1995. Dípteros muscóides da área urbana de Corumbá, Mato Grosso do Sul, Brasil. Revista Brasileira de Biologia 55:351-354.

Carvalho, C. J. B. DE; Moura, M. O. \& Ribeiro, P. B. 2002a. Chave para adultos de dípteros (Muscidae, Fanniidae, Anthomyiidae) associados ao ambiente humano no Brasil. Revista Brasileira de Entomologia 46(2):107-114

Carvalho, C. J. B. De; Couri, M. S.; Toma, R.; Rafael, J. A.; Harada, A. Y.; Bonatto, S. R.; Henriques, A. L. \& Gastal, H. A. De O. 2002 b. Principais coleções brasileiras de Diptera: histórico e situação atual. In: Costa, C.; Vanin, S. A.; Lobo, J. M. \& Melic, A. eds. Proyecto de Red Iberoamericana de Biogeografia y Entomologia Sistemática (PrIBES). Zaragoza, Monografias Tercer Milenio, SEA, vol. 2, p. 37-52.

Carvalho, C. J. B. De; Pont, A. C.; Couri, M. S. \& Pamplona, D. 2003. A catalogue of the Fanniidae (Diptera) of the Neotropical Region. Zootaxa 219:1-32.

Chillcott, J. G. 1961. A Revision of the Nearctic species of Fanniinae (Diptera: Muscidae). The Canadian Entomologist 92(Supl. 14):1-295.

CourI, M. S. 1992. Immature stages of Fannia pusio (Wiedemann, 1830) (Diptera, Fanniidae). Revista Brasileira de Biologia 52(1):83-91.

Gomes, P. R.; Koller, W. W.; Gomes, A.; Carvalho, C. J. B. De \& ZoRZATTO, J. R. 2002. Dípteros fanídeos vetores de ovos de Dermatobia hominis em Campo Grande, Mato Grosso do Sul. Pesquisa Veterinária Brasileira 22(3):114-118.

Wendt, L. D. \& Carvalho, C. J. B. DE. 2009. Taxonomia de Fanniidae do Sul do Brasil - II: Novas espécies e chave de identificação de Fannia Robineau-Desvoidy. Revista Brasileira de Entomologia 53(2):171-206. 